Théologiques

Théologiques

\title{
De la possibilité aléatoire mais promise d'une critique des traductions bibliques
}

\section{Alexis Nouss}

Volume 15, numéro 2, 2007

La traduction des textes sacrés

URI : https://id.erudit.org/iderudit/017772ar

DOI : https://doi.org/10.7202/017772ar

Aller au sommaire du numéro

\section{Éditeur(s)}

Faculté de théologie et de sciences des religions, Université de Montréal

\section{ISSN}

1188-7109 (imprimé)

1492-1413 (numérique)

\section{Découvrir la revue}

\section{Citer cet article}

Nouss, A. (2007). De la possibilité aléatoire mais promise d'une critique des traductions bibliques. Théologiques, 15(2), 47-66.

https://doi.org/10.7202/017772ar

\section{Résumé de l'article}

Le religieux et le sacré sont des notions qui réclament inévitablement leur rattachement à la sphère du transcendant dont la nature rend pour le moins complexe l'élaboration de critères d'évaluation quant aux pratiques inhérentes. Cet article analyse ainsi les obstacles qui se posent devant l'essai d'évaluation critique des traductions bibliques. Outre le niveau textuel et linguistique, toute évaluation doit d'emblée prendre en compte les dimensions théologiques latentes. Il importe de cerner le substrat théologique à l'oeuvre dans les positions traductologiques, en ce qui concerne le domaine biblique, de même que ce substrat agit dans les cultures sécularisées sous la forme d'un impensé ou d'un investissement de substitution.

Les grandes lignes d'une étude de ce type sont ici d’abord suggérées en envisageant les difficultés que pose l'examen des Bibles confessionnelles et militantes. Puis est abordée la divergence, dans les trois monothéismes, des attitudes traductionnelles en regard de la textualité révélée : judaïsme et islam se rejoignent dans la reconnaissance de la dimension interprétative, tandis que le christianisme vise à l'efficacité dans la transmission du message. Il est enfin fait état de la question du messianisme et de sa compréhension, qui pèse avec la même pertinence dans les orientations traductologiques.

La dernière partie procède à un retour sur la «nouvelle traduction » de la Bible parue chez Bayard/Médiaspaul, avant de conclure sur une défense du concept de « traduction athée » qui articulerait un principe d'altérité dont le texte biblique est le premier véhicule.
Tous droits réservés @ Faculté de théologie et de sciences des religions, Université de Montréal, 2007
Ce document est protégé par la loi sur le droit d'auteur. L’utilisation des services d'Érudit (y compris la reproduction) est assujettie à sa politique d'utilisation que vous pouvez consulter en ligne.

https://apropos.erudit.org/fr/usagers/politique-dutilisation/ 


\title{
De la possibilité aléatoire mais promise d'une critique des traductions bibliques
}

\author{
Alexis Nouss \\ Département de linguistique et de traduction \\ Université de Montréal
}

La traduction renvoie à la généalogie, puisqu'elle dévoile un engendrement, le sien, à partir de l'original. Enfant fidèle, rebelle, prodigue, renié ou adultérin, les bonheurs et malheurs de la condition parentale trouvent leurs équivalents dans le passage textuel interlinguistique. La traduction biblique y échappe moins qu'une autre dans la mesure où son contenu traite volontiers d'engendrements (les filiations — prophétiques, tribales, royales, sacerdotales et autres - dans l'Ancien Testament, la famille christique et la communauté des disciples dans le Nouveau ${ }^{1}$ ) et que, par ailleurs, la culture chrétienne adopte une telle optique pour penser le rapport entre les deux Testaments.

Paradoxalement, la traduction biblique, qui a fourni un cadre paradigmatique au développement de la théorie de la traduction en Occident, tant au niveau empirique par l'importance textuelle et langagière de ses accomplissements (la Septante, la Bible de Luther, la King James), qu'au niveau analytique par ses principes herméneutiques (depuis saint Augustin et saint Jérôme jusqu'à Schleiermacher et Benjamin), est celle qui est la plus capricieuse quant au jugement possible sur sa réussite généalogique, s'exposant à coup sûr à l'opprobre d'infalsifiabilité chère à l'argumentation de Karl Popper. Un problème spécifique et essentiel pour la traduction des textes sacrés ${ }^{2}$ est en effet celui de son évaluation. Il se pose également dans le domaine littéraire - seule la traduction des textes pragmatiques y échappant dans la mesure où la clarté et la précision des objectifs autorisent des procédures de validation explicites —, avec toutefois la possibilité de cerner

1. «Ancien Testament» et «Nouveau Testament»: en référence au lexique courant.

2. Les termes «sacré » de même que «religieux » ou « révélé » méritent une approche critique qui déborderait le cadre épistémologique de cet article et sont donc employés par commodité. 
les hésitations que soulèvent les variables du jugement esthétique en les rabattant sur des paramètres historiques et sociologiques auxquels invite l'immanence du champ considéré. Mais le religieux et le sacré sont des notions qui réclament inévitablement leur rattachement à la sphère du transcendant, dont la nature rend pour le moins complexe l'élaboration de critères d'évaluation.

Ainsi, la catégorie "non confessionnelle », avancée dans les typologies bibliques habituelles, par exemple dans le domaine francophone, pour qualifier les traductions de Dhorme ou de La Pléiade. Elle brouille l'exercice, car elle récuse toute neutralité. L'appartenance confessionnelle est-elle jugée positive ou négative? Dans le premier cas, les Bibles traduites selon un parti pris religieux particulier ne pourraient être comparées entre elles, en vertu du principe de tolérance, ni avec des traductions non confessionnelles, qui relèvent d'une démarche distincte. Ces dernières relèvent en effet d'une idéologie et l'examen de leurs stratégies de rédaction se heurte à cet obstacle rédhibitoire.

En admettant malgré tout la pertinence de la catégorisation, il demeure que toute évaluation doit prendre en compte la dimension théologique latente et ouvre ainsi à des analyses qui dépassent le simple niveau textuel. Une théologie de la foi confrontée à une théologie du savoir intellectuel, un théologisme ${ }^{3}$ juif opposé à un théologisme chrétien et, à l'intérieur de celuici, la divergence entre catholicisme, protestantisme et orthodoxie. Or, si le théologique a son mot à dire sur la traduction, sa légitimité et ses procédures, puisque le rapport entre le divin et l'humain procède en son principe d'une médiation (création, révélation ou transsubstantiation), soit il l'ignore parce que celle-ci lui est naturelle, soit il a tendance et intérêt à la dissimuler, parce qu'elle le constitue et qu'il ne veut avouer cette dépendance qu'il jugerait préjudiciable. C'est une position que l'on reconnaît aisément dans un christianisme refusant sa dette à l'égard du judaïsme.

Il n'est pas fortuit que la notion d'équivalence dynamique, par opposition à équivalence formelle, ait été pensée par un praticien de la traduction biblique et à son propos, dans le cadre d'un missionnarisme où l'intention de convaincre emporte toute l'entreprise (voir ci-après). Comment évaluer une traduction dont la soumission à un projet de conversion est patente et admise, ce qui guide l'ensemble des traductions confessionnelles? Reste le texte de la traduction dans son rapport même à ce qu'il

3. Ce terme sert à designer une théologie étroitement confessionnelle. 
véhicule, c'est-à-dire la logique de son fonctionnement interne: les rythmes et l'accentuation pour la Bible hébraïque, les registres de langue variés dans les différents évangiles, les degrés d'esthétisation contraires ou non aux sémantismes des versets.

Lorsque Walter Benjamin pose que «la traductibilité de certaines œuvres doit appartenir à leur essence » $(1997,15)$, les textes sacrés en fournissent l'illustration privilégiée, ainsi que le modèle, et il conclut son essai sur leur exemplarité. Jacques Derrida le formule radicalement: «L'à-traduire du texte sacré, sa pure traductibilité, voilà ce qui donnerait à la limite la mesure idéale de toute traduction. Le texte sacré assigne sa tâche au traducteur, et il est sacré en tant qu'il s'annonce comme traductible, simplement traductible, à-traduire...» $(1987,234)$. Le concept d' »appel à la traduction» (ibid.) résonne adéquatement avec les notions théologiques de $k o l$ ( « voix») dans le judaïsme, pneuma («souffle») dans le christianisme et $d h i k r$ ( «évocation») dans l'islam.

Or, comment juger de la réception d'un appel si ce n'est en examinant la justesse de la réponse ? À savoir que la réponse ne doit pas s'approprier la valeur de l'appel en le rendant obsolète par le fait qu'il a été entendu, mais qu'elle doit réaffirmer et maintenir sa pertinence en se faisant appel à son tour. C'est ce qu'exprimait Martin Buber ${ }^{4}$, lorsqu'il affirmait que la Bible était de part en part "message » (Botschaft), au sens d'enseignement divin, et que celui-ci se manifestait par un rythme, phonétique et structurel, infusant la textualité biblique et affectant le lecteur (1936; voir la traduction anglaise: 1994). Henri Meschonnic fera du rythme, dans un sens différent et plus large, le concept-clé de sa poétique du traduire et avertira: "Traduire n'est pas traduire si on ne rend pas le rythme qu'on a reçu » (2004, 213-218). Pour la traduction biblique, l'appel se dédouble: un appel destiné à ceux qui en sont les récipiendaires, à savoir les lecteurs, et un appel au texte sous la forme d'une incitation, reçue par ces derniers, à se rediriger vers le texte. Une traduction d'un texte sacré ne saurait réclamer toute l'attention pour elle-même au risque, dans la tradition monothéiste, de verser dans l'idolâtrie. D'où le risque pour une poétique traductive de verser en esthétique.

4. Martin Buber et Franz Rosenzweig apparaissent dans cet article à la fois pour leur traduction de la Bible hébraïque en allemand et pour leur œuvre théologique. 


\section{Traductions confessionnelles}

Les Bibles confessionnelles posent une série de problèmes qu'il importe d'énoncer. En premier lieu, la procédure d'évaluation en traduction consiste à remonter à la source et à examiner si les énoncés et les énonciations originaux retrouvent par correspondance ou recréation leurs spécificités dans la traduction, ce qui ne se restreint évidemment pas à la considération de l'auteur, d'une pertinence limitée et fragile, mais recourt à la prise en compte de tout son environnement historique, social, culturel et idéologique. Pour les Bibles confessionnelles qui reconnaissent le caractère de révélation du texte sacré, le cadre original échappe à l'investigation. Pour les autres, qui attribuent au texte une rédaction humaine, multiple et diachronique, la tâche se heurte aux limitations des connaissances, notamment quant aux anciennes cultures sémitiques.

En second lieu, les autorités ecclésiastiques admettent les retraductions, surtout lorsqu'est ignoré un principe d'autorité, tel celui attaché à la King James version, la Bible du Roi James (1611), quoique le domaine francophone puisse lui aussi revendiquer une version royale, la Bible de Charles $\mathrm{V}$, traduite pour le monarque par Raoul de Presles trois siècles auparavant (1377), mais dont l'impact n'eut que peu de conséquences. Ce qui soulève une situation paradoxale où la dimension historique est reconnue dans sa nécessité, justifiant les retraductions confessionnelles, alors que la foi menant ces entreprises transcende pour sa part l'inscription chronologique. Dans le cadre d'une confession donnée, il paraît délicat de juger une profession de foi supérieure à une autre. Comment d'ailleurs rivaliser avec les traducteurs occupant la plus haute place dans la hiérarchie? Saint Jérôme - à tout seigneur tout honneur —, saint Mesrob Machtots, inventeur de l'alphabet arménien et traducteur de la Bible en cette langue au $\mathrm{V}^{\mathrm{e}}$ siècle, un siècle après le premier, Cyrille et Méthode qui la traduisirent en slavon au $\mathrm{IX}^{\mathrm{e}}$ siècle?

En outre, les confessions participant de la filiation monothéiste n'adoptent sur cette question que peu de tolérance. Les catholiques ont leur Bible, les protestants la leur et la TOB (Traduction cecuménique de la Bible), si elle est louée pour sa vertu spirituelle de réconciliation, n'est pas utilisée dans la pratique en raison de son niveau stylistique trop soutenu ou de son aspect érudit gênant, dans le cadre d'une lecture paroissiale.

À quatre siècles de distance, une visée traductive similaire semble mener au niveau linguistique les entreprises de Luther et de Buber et Rosenzweig. Le premier affirme avoir été à l'écoute de la langue des maisonnées et des 
rues, les seconds privilégient le terme verdeutschen («rendre en allemand » ou "germaniser») sur celui übersetzen pour qualifier leur travail, comme en témoigne le titre du recueil d'essais publié en 1936, Die Schrift und ibre Verdeutschung. Les deux entreprises sont également placées par leurs auteurs dans la dimension historique de leur actualité. Luther combat les dérives du catholicisme romain et, très rapidement, en vient à s'opposer à la souveraineté politique de Charles Quint, ce qui entraînera des luttes sanglantes au sein du Saint Empire. Buber et Rosenzweig commencent leur traduction en réponse au mouvement visant à purger la culture allemande des influences juives, né dès avant la Première Guerre mondiale et poussé à son paroxysme par le nazisme. Tous ces éléments n'empêchent pas un éminent professeur en études allemandes de juger la traduction de Luther (XVI ${ }^{\mathrm{e}}$ siècle) acceptable et la seconde (XXe siècle) illisible (Snyder 1995, 36).

Enfin, sans quitter la question de la foi, l'intégrité et l'intégralité sembleraient devoir imposer leur exigence, précisée par le doublet lexical, à la réception d'un texte auquel la croyance religieuse attribue une source divine. Pourtant, en matière de traduction chrétienne, le souci disparaît des consciences et se multiplient traductions partielles ou traductions indirectes (à partir du latin autant que de quelques langues occidentales). S'il n'est pas du ressort d'une critique traductologique d'en évaluer le déficit d'un point de vue théologique, dans son propre domaine elle est bien en peine de procéder à un quelconque examen devant ce type de traductions qui, dans une certaine mesure, se disqualifient d'emblée.

De même, l'examen des traductions bibliques se fait à l'intérieur d'une orientation confessionnelle ${ }^{5}$, sans que - ou fort rarement - soit entreprise une critique comparée entre Bibles juives et chrétiennes, par exemple, ou encore en adoptant le monothéisme comme cadre référentiel entre Bible et Coran, alors que les trois textes sacrés ont initié de nombreuses lectures les confrontant, à des degrés de neutralité scientifique variés, et qu'existe en français la somme traductive d'André Chouraqui regroupant Ancien Testament, Nouveau Testament et Coran. Il est permis de considérer l'élaboration d'une critique de la traduction des textes sacrés, de nature comparatiste, qui déborderait le cadre monothéiste et étudierait les enjeux, principes et méthodes des procédures traductives, quelles qu'en soient les

5. Mentionnons, par exemple, Long (2005) ou Auwers et ses collaborateurs (2002), qui traitent des Bibles juives dans une vague catégorie «autres". La Routledge Encyclopedia of Translation Studies (2001; voir ci-après) consacre trois entrées séparées à la traduction de la Bible, de la Torah et du Coran. 
sources formelles (orale ou écrite, voire langagière ou plastique ${ }^{6}$ ) ou spirituelles (des polythéismes aux monothéismes, en passant par les animismes et chamanismes) $)^{7}$.

L'affirmation explicite d'une direction traductive n'implique toutefois pas que le texte n'offre aucun intérêt en dehors de son efficacité rhétorique, puisque toute orientation militante doit choisir des stratégies qui, en tant que telles, sont susceptibles d'attention. Ainsi de la récente traduction dite féministe en anglais par Mary Phil Korsak (1992), dont l'option littéraliste permet de resémantiser avec justesse le texte biblique; ainsi du nom de la première femme dont sa version reprend, en l'expliquant, la forme hébraïque, "Hawwa [Khava]», pour en faire ressortir l'étymon khaï, la vie, que souligne la suite du verset: "car elle a été la mère de tout ce qui vit» (Gn 3,20). Au demeurant, le littéralisme, entre autres sur cet exemple, accompagnait déjà un siècle plus tôt The Woman's Bible d'Elisabeth Cady Stanton, qui s'appuyait sur une version antérieure, elle aussi littéraliste, d'une traductrice évangéliste, Julia Evelina Smith (voir Flotow 2000). En revanche, les initiatives contemporaines, affichant une sensibilité similaire aux traductions gender-neutral ou gender-inclusive, telles que la New International Version de l'International Bible Society, n'offrent qu'un faible intérêt sur le plan traductologique, car elles ne se soucient guère de l'original et, portant leur effort sur l'usage coupable de termes révélateurs de valeurs patriarcales, androcentriques, voire misogynes, procèdent par rapiéçage lexical, substituant «person" ou «anyone " à «man", "child» à «son ", «parent» à «father» ou «brother» et, pour les plus extrêmes, «God the father-mother" à "God the father».

6. Cette dernière option a été suivie dans le recueil Translating Religious Texts (Jasper 1993) qui, sur huit articles, en consacre deux au domaine pictural. La croyance n'ignorant pas les procédures de l'inconscient et celui-ci pratiquant volontiers l'interaction des mots et des images, il paraît normal de soumettre les deux champs à l'examen.

7. Le projet du présent numéro de Théologiques s'inscrit, à un niveau très modeste, dans cette inspiration qui influença le choix d'ouvrir le corpus des textes sacrés aux traditions bouddhiste et amérindienne et de soumettre à tous les participants un questionnaire destiné à assurer la cohérence de leurs réflexions. Celui-ci se lisait comme suit : 1) Le message religieux est-il favorable à la traduction? 2) Existe-t-il une ou des traductions qui se soient imposées comme canoniques (au sein d'une histoire des traductions marquantes) ? 3) Qui traduit? Pour qui ? Sous quelle autorité ? 4) La traduction «scientifique" est-elle admise, voire encouragée ? 5) Quelles sont les principales difficultés de traduction? 


\section{Les trois monothéismes}

Mais si la traductologie tire une partie majeure de ses origines dans la traduction du texte sacré, il semble qu'elle gère malaisément son héritage, à moins qu'il ne faille admettre qu'elle cherche à en refouler les composantes non conformes à une certaine ligne idéologique. C'est d'emblée le privilège accordé à la Bible qu'il convient d'interroger et à une Bible bien ciblée, car le signifiant «Bible », dans son singulier qui efface un pluriel grec ( $t a$ biblia, "les livres »), lui-même reprenant un pluriel hébreu (ha-sefarim) désignant l'ensemble (36 ou 24, selon les décomptes possibles) des livres constituant le $\operatorname{Tanakh}^{8}$, renvoie au christianisme dans son ambition œcuménique. En outre, la traductologie, en tant que discours et savoir universitairement régentés, est née en Occident et elle endosse l'oubli que ce dernier, dans son autodéfinition, a soigneusement perpétué, du rôle de la culture arabomusulmane portée par le Coran. L'hellénisme de la tradition occidentale doit en partie sa permanence, non seulement à l'action concrète des traducteurs du monde arabo-musulman, véritables passeurs entre le grec et le latin, mais non moins au dialogue spéculatif que ses penseurs ont entretenu avec les philosophes grecs, qui donna le kalam et l'œuvre de Ibn Rushd (Averroès).

La rupture monothéiste, en tant que telle, entraîna une radicale transformation de la dimension traductionnelle du religieux. Dans l'Antiquité, les divinités circulent d'une culture à l'autre, se traduisent d'une croyance à l'autre, par exemple entre l'Égypte et la Grèce ou entre Athènes et Rome, en une théâtralité métaphysique à laquelle assistent les humains, sans qu'il ne leur soit demandé de participer à cette économie transcendante, qu'ils intègrent à leur gré dans la défense de leurs propres intérêts en dehors des querelles de chapelles. À cette traductibilité horizontale, le monothéisme va substituer un axe vertical où le divin exhorte l'humain à traduire sur la scène de son histoire les exigences de la révélation. Le sujet $(\mathrm{du})$ religieux n'est plus un spectateur passif, mais devient l'acteur de dramaturgies passant rapidement au stade de rivales.

Dans le cadre global de cette nouvelle distribution des rôles adaptée au scénario civilisationnel inédit que l'Occident se choisit à partir des livres sacrés à sa disposition, des différences apparaissent. Pour les cerner, il est pertinent de comparer la désignation «interne» des trois textes fondateurs et d'en ressortir trois attitudes distinctes: mikra, la lecture (publique, en

8. Voir la note suivante. 
assemblée) ${ }^{9}$; Coran, l'appel (ou la lecture); Évangile, la bonne nouvelle. Les deux premières demandent l'effort d'une réception, la dernière, une simple acceptation, dessinant ainsi deux modalités traductionnelles: l'une reconnaît le crible nécessaire d'une interprétation dont la dimension humaine garantira la multiplicité des sens possibles, la seconde vise la seule transmission du message. Ces deux attitudes face à la traduction trouvent leurs pendants dans des procédures différentes sur le plan de l'herméneutique ou du dispositif institutionnel : judaïsme et islam, d'une part, accolent au texte fondateur les appareillages exégétiques que sont le Talmud ou les hadits - accordant au commentaire la même inspiration divine que la Torah et le Coran - et, d'autre part, refusent l'instauration d'un clergé comme instance médiatrice et organisatrice de la socialité religieuse. Le geste traductif n'est pas l'apanage d'un groupe social, puisqu'il s'offre à tous dès le stade de la révélation, car « un jour, et nul ne saura plus tard quel jour, jaillit à travers cette langue originaire du genre humain la langue de l'humanité en l'homme, la langue du verbe» (Rosenzweig 1998, 89). Les trois textes sacrés opèrent pour les trois religions comme des matrices ${ }^{10}$ identitaires et culturelles, comme des sites recueillant à la fois la fabrique et le secret de la genèse du monde et de l'humain, mais dans ce dispositif elles comprennent et traitent différemment le problème de l'origine (voir Sibony 1997) et, conséquemment — vu la nature textuelle d'une telle matricialité - la problématique de l'original, le statut de ce qui est à-traduire. D'une certaine manière, le processus de l'incarnation que le christianisme lie au corps du Christ est pour le judaïsme et l'islam dirigé vers le «Livre ", qui devient réceptacle de ce que la théologie chrétienne nomme "consubstantialité ». D'où l'impossibilité de canoniser une traduction comme cela s'est produit dans la chrétienté.

Or, de la diversité des pensées de la traduction quant aux textes révélés, la traductologie est peu soucieuse. L'article «Bible Translation» de la Routledge Encyclopedia of Translation Studies, ouvrage de référence, commence tranquillement par l'énoncé: "The Bible is the holy book of Christianity» (Baker 2001, 22). Exit le judaïsme, familier des exodes et expulsions, qui ne pâtira guère d'une éviction supplémentaire. Le manque de rigueur est patent mais non étonnant, puisque l'auteur de l'entrée est

9. Une des deux désignations usuelles, l'autre étant strictement descriptive et acronymique: TaNaKH, pour Torah («Enseignement»), Neviim («Prophètes»), Ketouvim ( «Écrits»).

10. Les signifiants «matrice» et « livre» sont joints dans la sourate coranique 43,3 . 
Eugen Nida, figure éminente de l'Alliance biblique universelle et théoricien devenu incontournable, à qui l'on doit la distinction entre formal equivalence et dynamic (ou functional) equivalence, élégante resucée aux couleurs linguistiques de l'antique opposition entre le sens et la forme, dont les manuels traductologiques gomment soigneusement ce qui la fonde, à savoir la motivation missionnaire de conversion par la diffusion des Saintes Écritures. Il demeure plus que discutable, pour les responsables de l'encyclopédie, d'avoir demandé la rédaction de l'entrée en question à un spécialiste dont le travail, parfaitement respectable en soi, se place néanmoins sous le signe du prosélytisme. L'analogie serait de confier à un membre du bureau central du Parti communiste - s'il en reste — la rédaction d'une notice sur le marxisme dans un ouvrage qui se voudrait de nature scientifique.

\section{Substrat théologique}

De tels impairs ne doivent pas empêcher d'avancer l'existence d'un substrat théologique dans les positions traductologiques, de même qu'il est à l'œuvre dans les cultures sécularisées, sous la forme d'un impensé dans les développements de leurs philosophies ou d'un investissement de substitution, la langue par exemple. Que les théologies monothéistes viennent éclairer la compréhension du geste traductif paraît naturel puisqu'elles s'appuient sur une instance langagière considérée comme fondatrice — le "Dieu dit ...» des premiers versets de la Genèse, le «Verbe fait chair» du prologue de l'Évangile de Jean, le Dieu au calame de la sourate 96. Mais au-delà de ce constat, il s'agit d'examiner de quelle manière chacune d'entre elles détermine l'articulation entre un tel influx divin et la responsabilité humaine, dégageant sur cette base un jugement traductionnel. Le rapport au texte révélé se mêle évidemment au rapport avec la langue de la révélation et le degré de sacralisation dont elle est investie, ce qui incite Jean-René Ladmiral $(1990,131)$ à proposer le tableau suivant:

En schématisant, il serait possible de figurer un axe linéaire à l'un des pôles duquel on trouverait le Coran, où la sacralisation du texte original et de la langue-source est totale: ce serait la position extrême, où la traduction fait problème; à l'extrême opposé, le pôle où la "sacralisation » du texte-source est elle-même problématique et où, surtout, il n'y a pas de langue de Dieu, serait la version catholique de la traduction biblique. Entre ces deux extrema, on trouverait respectivement les versions juive et protestante de ladite traduction biblique. 
En déplaçant l'interrogation sur la pratique même et en passant du théologique au religieux, on constate que les trois monothéismes n'adoptent pas une même attitude en regard de la traduction, puisqu'en contraste avec le christianisme, judaïsme et islam intègrent dans leur dispositif d'institution la possibilité et l'empiricité de la traduction. Le livre de Néhémie, qui narre le retour des exilés babyloniens en Judée, rapporte l'épisode de la lecture par le prophète Ezra, prêtre et scribe, de la "Torah de Moïse » devant tout le peuple réuni à Jérusalem et précise: "les Lévites faisaient comprendre au peuple la Torah, le peuple étant debout. Et ils lisaient dans le livre, dans la Torah de Dieu, avec clarté et de manière intelligible, et eux comprenaient la lecture " (Néhémie 8,7-8). Ceci signifie pour l'exégèse que la lecture s'accompagnait de traductions et de commentaires. Les sourates dites « de Joseph» et «des ornements » associent la révélation et la langue arabe, soulevant d'emblée la question de la traductibilité, à la fois quant à sa légitimité, sa possibilité et sa nécessité: " 1 . Ce sont les signes ${ }^{11}$ du livre évident. 2. Nous l'avons fait descendre du ciel en langue arabe, afin que vous le compreniez » (sourate 12); "1. J'en jure par le livre évident. 2. Nous l'avons envoyé en langue arabe, afin que vos le compreniez» (sourate XLVIII, trad. Kasimirski 1970). Sur le plan pratique, les éditions courantes de la Torah intègrent en marge des versets le targum, leur traduction en araméen provenant des anciennes lectures synagogales publiques et, depuis 1936, les autorités islamiques permettent la publication de traductions à condition qu'elles apparaissent parallèlement au texte original. Pour le christianisme, la traduction ne se pose pas en relais de la révélation, puisqu'elle opère au principe de son développement. Diverses sources écrites en hébreu et en araméen passent en grec chez les évangélistes par le crible de l'interprétation et d'une altération adoptée en fonction du message religieux à diffuser. La Bible en grec des Septante fournit pour les premières Églises chrétiennes le texte de l'Ancien Testament, puis est remplacée en Occident - l'orthodoxie de liturgie grecque la conserve - par une autre traduction: la Vulgate en latin de Saint-Jérôme, qui ouvre la voie d'une clarification de l'accès aux Écritures, cependant qu'un large corpus exégétique se développait. Sans omettre qu'au niveau historico-politique, une forme de traduction présida à la propagation et au succès exponentiel de la nouvelle religion, à savoir la conversion de l'empereur Constantin, sans

11. Ayia désignant en arabe à la fois le signe et le verset, ce terme pose d'emblée la concomitance de la lecture et de l'interprétation. Il signifie également miracle, troisième acception permettant d'articuler la transcendance et la finitude. 
laquelle — et malgré quelques atermoiements ultérieurs — l'enseignement de Jésus serait demeuré l'apanage d'une secte sémitique. Au demeurant, même le rabbi de Nazareth qui parlait araméen, comme son entourage, ne s'exprime pas en version originale et bénéficie, par les Évangiles, d'un parfait doublage dans les langues d'Athènes puis de Rome. Selon Willis Barstone, qui emprunte son imagerie à la mythologie comme à la philosophie, le processus de traduction dans le christianisme s'opère sous le double patronage de "Protée transformant, invisible, les vagues et d'Héraclite plongeant sa jambe dans des fleuves toujours changeant » (1993, 175, ma traduction).

Les métaphores récurrentes du serviteur, du miroir ou de la fausse monnaie ont clairement illustré la conception platonisante et négative, qui fait de la traduction une copie de l'original, considération dont l'influence la plus manifeste apparaît dans la première législation française sur la traduction, puisque celle-ci alignait la juridiction concernant la traduction sur celle traitant de la contrefaçon. Dans le destin des textes sacrés, la copie exerce une fonction primordiale, puisque ceux-ci se constituent progressivement jusqu'à leur fixation canonique, à partir de textes divers, eux-mêmes ayant recueilli des sources orales, transmises au fil des générations. En ce sens, la copie est translation au sens matériel avant de se prêter à la traduction. Or, en tant que thème, elle a été déclinée, dans le discours théologique, sous la forme du principe d'imitation que judaïsme et christianisme ont particulièrement développé, mais avec une différence majeure qui tient au niveau auquel demeure l'instance à imiter (voir Buber 2003). Pour le christianisme, l'imitatio Christi s'inscrit dans la finitude d'une vie humaine, ou du moins humanisée, et dans la spécificité de cette vie-là, qui condense les aspects attendus d'une imitatio Dei. Un signifié, un signifiant. Ou encore: une traduction prendra en compte les médiations qui ont formalisé le message. Le judaïsme ne peut s'appuyer sur l'intervention d'une figure, fût-elle divinisée, mais, en revanche, il met de l'avant la création de l'humain à la ressemblance et à l'image de Dieu (Gn 1,26). La similarité est déposée en germe dans chaque créature, charge lui en étant donnée de la développer dans sa propre histoire, ce qui pourra s'effectuer en suivant la seule donnée que l'humain possède dans sa connaissance du divin, à savoir son agir dans le monde. Deux options ainsi dégagées: un texte est écrit, il faut le traduire; un texte s'est écrit, il faut en traduire la manière.

Le messianisme trace une autre ligne de partage quant au paradigme traductif, dans la mesure où le souci d'une césure temporelle, selon qu'elle 
soit passée ou future, clôture ou ouverture, dessine deux directions traductionnelles: Jésus reconnu comme le Christ ou Mahomet comme le "sceau des prophètes ", l'ultime jalon de la révélation, orientent l'histoire de l'humanité en un sens qui n'est pas celui du judaïsme dont l'attente du messie constitue un principe théologique majeur. L'à-venir peut se comprendre comme un à-traduire, l'advenu comme un à-retraduire. C'est encore une autre perspective qu'ordonne le destin final des figures fondatrices: Moïse meurt comme tout humain, mais le lieu de sa sépulture demeure inconnu; Jésus ressuscité monte au ciel; Mahomet s'éteint dans la maison d'Aïcha puis est enterré près de la mosquée de Médine. Les trois "origineurs » ou plutôt leurs trois corps absents suggèrent trois attitudes en regard du textesource, selon qu'il sera considéré comme en dehors de l'histoire (christianisme), en retrait de l'histoire (judaïsme), ou dans l'histoire (islam). Ce sont trois modalités du rapport traductif au sens qui se dégagent et qui correspondent à trois degrés de sacralisation de la langue originale: le primat du sens sur la forme (le dehors de l'histoire), un équilibre entre sens et forme (le retrait), le primat de la forme sur le sens (le dedans).

Nulle intention de suggérer que la pratique traductive attachée à telle ou telle spiritualité suivra obligatoirement le modèle dégagé en fonction de leurs contenus théologiques ou mytho-narratifs, encore moins de les classer par ordre de préférence - les excès du littéralisme ou de l'adaptation, autant que les réussites existent dans les trois traditions - puisqu'il ne s'est agi que de développer des éclairages sur le geste traductif à partir de ces contenus et qu'au demeurant les découpages prémentionnés selon différentes lignes de séparation ne se confondent aucunement. Sur le plan de la pratique, les usages sont variés - le rendu traductif de Dhorme n'est pas celui de Segond et la poétique de Chouraqui diverge de celle de Meschonnic - et, le reconnaissant, la réflexion traductologique gagne à dresser un tableau pluriel des visées traductives que peuvent inspirer les données théologiques des trois monothéismes et à repérer leurs influences sur les courants théoriques. En négligeant les autres options «théotraductionnelles » pour ne retenir que celle du christianisme ${ }^{12}$, la traductologie s'est pliée à sa tendance néologisante (au sens large: instauration d'un nouveau logos) qui, naturalisant la traduction, tend à faire disparaître l'original. Par une

12. Plus encore dans la ligne doctrinale de l'ascétisme médiéval, prônant la renonciation aux préférences personnelles et aux plaisirs de la sensualité textuelle, défendant l'abstraction d'un sens décorporalisé et idéologiquement contrôlé, selon Douglas Robinson $(1992,6)$. 
stratégie qui, en tant que telle, cherche d'abord à nourrir son souci d'efficacité, le besoin idéologique d'une discipline langagière est venu se combler auprès du discours théologique dominant dans la société qui a vu son émergence. S'appuyant sur une vision dualiste de la langue et privilégiant le signifié, cette pensée traductologique ne pouvait trouver meilleure légitimation que dans l'effet «Verus Israel », le principe chrétien de la "préfiguration, ce paradigme théologique du signe» (Meschonnic 2004, 113) : le judaïsme n'a eu pour rôle que d'annoncer la venue du Christ, de même que le signifiant (la forme) ne sert qu'à introduire au signifié (le sens). Pour ce qui est du corpus biblique, la terminologie et l'archivistique permettent de constater les glissements à cet effet: le Tanakb se transforme en Ancien Testament, la Bible juive devient le texte de la Septante, alors que celle-ci inclut ce que le canon hébraïque considère comme apocryphe, et la mikra (lecture) cède la place aux Écritures.

\section{Une nouvelle Bible?}

Une parfaite illustration en sera fournie par un bref réexamen de la récente «nouvelle traduction» de la Bible parue chez Bayard/Médiaspaul. Pour la présenter, Frédéric Boyer, maître d'œuvre de l'entreprise, écrivait: "La Bible, telle que notre culture l'a façonnée et nous l'a transmise, est un formidable "disque dur» qui a accumulé la mémoire des langues et des littératures qui l'ont écrite et traduite ${ }^{13}$. Pluralisme des sources, des textes et des versions érigé en postulat, guidant toute l'entreprise. À ce disque dur, toutefois, il est par exemple loisible d'opposer un noyau pareillement dur ${ }^{14}$, le texte du Tanakh dans sa matérialité scripturaire qui se retrouve dissimulée dans les plis d'une textualité aux contours flous et dont l'option traductologique choisie effacera la poétique spécifique. Une «bible» donc, avec l'initiale en minuscule, audace typographique qui n'est pas allée jusqu'à la restauration d'un pluriel qui n'est reconnu que dans l'insistance sur la «polyphonie» du texte biblique. En laissant les sociologues de la culture s'interroger sur ce retour du religieux qui n'hésita pas, en 2001, à utiliser les canaux publicitaires les plus profanes, il importe de rappeler qu'une traduction de la Bible en français paraît tous les cinq ou dix ans en

13. Dossier de presse, brochure de présentation, p. 4.

14. En empruntant l'expression à George Steiner $(2003,7)$ qui l'utilise pour évoquer le refus du judaïsme de se traduire en une nouvelle spiritualité lorsqu'il a rejeté la messianité de Jésus. 
moyenne, le français étant la langue comptant le plus de traductions différentes. Depuis la nouvelle traduction Bayard/Médiaspaul ont été publiées, à titre indicatif, la Nouvelle Bible Segond (2002, la première édition datant de 1880) — dont l'importance aurait pu être saluée avec le même éclat et la Bible expliquée (2004), reprenant la Bible en français courant (1982, 1997).

Contrairement à l'argumentaire des vendeurs de traduction nouvelle, à partir de la première impression de la Bible par Gutenberg, jusqu'à la Bible de Lemaistre de Sacy, la Bible de Port-Royal et celles des siècles suivants, le catholicisme n'a cessé de diffuser en traduction son texte fondateur, notamment en France où l'on ignora le Concile de Trente. Sans compter les traductions protestantes et les tentatives en marge de l'orthodoxie, telle, au XVI ${ }^{\mathrm{e}}$ siècle, La Bible nouvellement translatée de Sébastien Castellion ${ }^{15}$, proche de Rabelais et Ronsard, destinée au peuple - celui que sa préface désigne sans péjoration comme les "idiots ", quatre siècles avant que ne paraissent The Complete Idiot's Guide to the Bible et The Bible for Dummies, ainsi qu'en France une Bible pour les nuls, sortie en 2004 et vendue à plusieurs dizaines de milliers d'exemplaires.

Dans la mesure où les arguments sur la plus grande authenticité ou justesse traductive peuvent être avancés par d'autres entreprises, c'est sans doute l'ambition esthétique ${ }^{16}$, qualifiée de nécessaire en référence aux "révolutions successives du langage littéraire et poétique du $\mathrm{XX}^{\mathrm{e}}$ siècle » (Boyer 2001, 23), qui motiva et autorisa un tel tapage médiatique. Il s'agit bien en ce sens d'une Bible catholique, comme sont catholiques l'autorisation des images et la magnificence des lieux de culte, bref la revendication du beau pour servir la foi. Un travail sur la langue à l'opposé de la démarche de Chouraqui. Pour reprendre des images du récit de la création, la Bible de Chouraqui et son recours à l'archaïsme offrent un texte lourd comme le limon primordial, tandis que la "traduction nouvelle » vise à la vivacité de l'écume ou du souffle, l'élan de la littérature.

Il faut admettre la réussite de la facture éditoriale: une présentation pertinemment allégée, des introductions et notes pour chaque livre, deux glossaires, précieux outils de référence, deux index. La nouveauté, toutefois, réside surtout dans la méthodologie adoptée, dite "du binôme ", qui invitait à s'associer, pour chaque livre, un exégète et un écrivain. Tous deux sont baptisés traducteurs dans le projet alors que, techniquement, aucun ne le

15. Rééditée en 2005 chez Bayard.

16. La présente analyse ne s'arrête qu'aux intentions du projet et ne rentre pas dans le détail d'un examen critique des résultats. 
soit: le second, sans accès à la langue originale, réécrivait le matériau brut ou littéral, scientifiquement annoté, fourni par le premier. Si les deux phases, décryptage et rédaction, sont familières de tout traducteur, ce qui fait la traduction tient à l'unité du sujet opérant. La dichotomie reflète et reconduit ce qui hante la métaphysique occidentale et que lui lègue le double héritage du platonisme et du christianisme: la séparation de l'esprit et de la lettre, du sens et de la forme, du signifié et du signifiant, césure pourtant véhémentement dénoncée dans la traductologie contemporaine. De surcroît, la pratique qui amène, il est vrai, certains renouvellements lexicaux heureux occulte toutefois de facto l'hébreu, l'araméen ou le grec, bref la présence de l'étranger, et aboutit à la traduction d'une traduction.

Les deux partenaires s'entendaient finalement par consensus, nous dit-on, sans que soit précisé sur quels principes et sans que, de leur propre aveu, pour les écrivains ne se soit posée la question de la foi. Certes, dans notre horizon post-structuraliste, tout texte n'est qu'un texte, mais on ne peut mettre entre parenthèses ce qu'une société en fait. Shakespeare n'est pas Salomon et Dante n'est pas Daniel. Par ailleurs, à quel titre faire appel à un écrivain? Un sacré en remplace un autre, le religieux par le littéraire, tant pis si le mythe homérique ou hugolien d'une inspiration artistique quasidivine date fâcheusement. Opérant la désacralisation d'un texte jugé figé dans ses versions antérieures en l'accompagnant d'une resacralisation par la grâce d'un pouvoir attribué à l'esthétique littéraire, la postmodernité s'avère bien plus dévote que la modernité. Lorsque Claudel traduisait les Psaumes ou que Pierre Emmanuel s'associait à l'entreprise de la Bible de Jérusalem, leur démarche prenait en compte l'infléchissement que le texte biblique impose à toute expressivité. Mais ceux-là étaient croyants et dépareraient dans la galerie terriblement contemporaine où figure une grande part des écrivains recrutés.

Nonobstant la contradiction entre l'annonce du projet global d'une «nouvelle traduction" et l'autonomie revendiquée de chaque livre traduit, la diversité rédactionnelle du texte biblique, due notamment à sa diachronie, pourrait justifier une diversité des styles traductifs, à condition d'oublier l'harmonisation nécessaire à son intégration dans une tradition religieuse où l'unité rhétorique fait pendant à l'unité transcendante de la source. Veut-on faire de cette Bible un objet seulement culturel - ce qui expliquerait l'usage de la minuscule initiale? Il s'agit cependant d'une traduction mise en chantier par des maisons d'éditions catholiques, qui ont de surcroît demandé une légitimation auprès des autorités concernées. Au demeurant, l'uniformatisation se réintroduit puisqu'un même écrivain va signer différents textes, la 
fougue d'un Osée et le lyrisme du psalmiste s'unifiant par exemple dans une même trivialité.

Quant à l'intertextualité existant entre les corpus bibliques juif et chrétien, puisque, théologiquement, le second s'appuie sur le premier et que, formellement, le corpus chrétien cite le corpus juif, la cohérence minimale n'est guère ici respectée. À cet égard, on ne peut que constater la bien plus grande liberté d'adaptation dans le corpus hébraïque, que ne sauraient justifier des considérations linguistiques. De même, l'index correspondant est cinq fois plus mince alors que la masse textuelle est dans la même proportion plus importante.

L'insistance sur la force de la forme littéraire révèle un souci d'esthétisation, écho d'une théologie de l'incarnation, qui engage aussi un parti pris sur la traduction. Mais l'exemple de la Bible Bayard ouvre sur une question plus large que nous abordons en matière de conclusion. Les textes sacrés initieraient donc deux attitudes: au-delà de la distinction première entre une traduction-écriture et une traduction-lecture où se reconnaîtraient davantage traditions juive et musulmane que chrétienne, s'opposent les deux pôles d'une traduction "auctoriale» et d'une traduction «traductoriale» qui délimitent l'espace des possibles traductifs.

\section{Pour une traduction athée}

Face au texte dit révélé, il faut révéler le texte, ce qui implique en quelque sorte l'effacement du traducteur. Le point demande précision, car tout un courant traductologique mène le combat pour rétablir précisément la présence du traducteur, la reconnaissance de sa médiation et la légitimation de ses interventions. À juste titre sur les plans sociologique et économique, car en ces domaines comme en d'autres, il importe de dénoncer toute forme d'aliénation et d'exploitation. Une confusion, toutefois, s'est installée qui comprend l'attention au signifiant professée par le littéralisme - ou ce qui est désigné comme tel — avec une "survisibilité » nécessaire et appliquée du traducteur, inversant le primat du sens en primat de la forme, alors que c'est le dualisme idéologique du signe qui doit être déconstruit, au profit de la reconnaissance du "continu du faire, de l'agir dans et par le langage ${ }^{17}$ » . Prétendre faire du traducteur un (simple) auteur, loin de le valoriser, érode

17. Meschonnic 1999, 24. Un des apports majeurs de son œuvre est d'avoir montré que la traduction biblique est le lieu privilégié pour mener ce combat, car «la Bible [hébraïque] est la seule aventure dans l'histoire du langage qui impose de penser autrement, et d'entendre ce que le signe ne sait pas entendre» $(2004,182)$. 
son statut car, en toute rigueur, il ne sera jamais qu'un second auteur, alors qu'il doit se vouloir un auteur second et qu'en outre la spécificité scripturaire de la traduction est de montrer que cette dernière catégorisation n'est pas minorisante, que la secondarité ne se colore de négativité que dans une idéologie du fondement contraire au devenir historique et portant les risques du fondamentalisme. Les théologies juive et musulmane du texte, considérant la forme même de ce dernier, suggèrent une vue contraire en rappelant que la Genèse commence par la lettre beth, deuxième de l'alphabet hébraïque, tout comme les traités talmudiques s'ouvrent tous à la page 2 et que la suite des sourates du Coran — dans l'ordonnance fixée et adoptée depuis le troisième califat — ne correspond pas à l'ordre historique de leur révélation. On ne lit donc qu'un texte second, en une invitation qui ruine toute prétention à la connaissance et donc à la possession du message authentique. L'origine est perdue dans un flou originaire.

Le corpus des textes religieusement fondateurs amène ainsi à proposer le concept de traduction athée qui se profile sur le modèle d'une écriture athée, c'est-à-dire non personnifiée, dont l'analyse a précisément été élaborée par Henri Atlan à propos de ces textes:

«la parole de Dieu» ne peut être intelligible qu'indépendamment des idées que l'on peut se faire, a priori, sur la personne de l'auteur. Ceci n'est d'ailleurs pas une particularité de la Bible. Il en est ainsi de tous ces textes particuliers, dits «sacrés» ou révélés, qui jouent un rôle spécial non seulement dans les religions dites "révélées", mais dans toutes les grandes traditions, notamment extrême-orientales. Comme dans les cas des Vedas et Upanishads, ces grands textes n'ont pas besoin d'auteurs pour être sacrés. Au contraire. C'est cette absence d'auteur identifié qui permet de les considérer comme différents des autres, pérennes sinon "éternels ", donnés uniquement pour être interprétés. $(2003,412)$

Ou pour être traduits. L'athéisme est une option conceptuelle disponible chez Rosenzweig comme chez Lévinas pour approcher un rapport nonidolâtre au divin, dont l'irréductibilité éclaire horizontalement la relation à autrui, visée pour laquelle le geste traductif est exemplaire. Toute théologie, au demeurant, peut s'appréhender comme une manière de traiter (avec) l'Autre et chaque texte sacré en écrit la charte. Dès lors, la traduction d'un texte sacré met en acte pour le sujet traducteur la présence de l'altérité et à l'altérité. La séparation que les théologies du monothéisme situent au principe de la création se retrouve dans la césure constituante (Spaltung) que le regard psychanalytique nous a habitués à reconnaître dans l'être de 
parole qu'est l'humain. Dès lors, s'ouvre la possibilité d'une évaluation des traductions bibliques qui s'opérerait à la lumière de ce principe d'altérité et s'attacherait aux modalités selon lesquelles il est retransmis au lecteur. Le texte biblique est porteur d'une promesse, celle d'un ici-bas ou d'un audelà aux couleurs d'un monde meilleur. Mais il est aussi porté par une promesse : que sa réception mène sur la voie de la première. Une telle promesse guide à la fois la traduction biblique et sa possible évaluation. Ce fut peutêtre cette conscience qui poussa en 1961 Gershom Scholem à discerner dans le projet de traduction de la Bible de Buber et Rosenzweig, en regard de ce qu'il advint du judaïsme allemand, « une part d'espérance» $(1974,446)$.

\section{Références}

Atlan, H. (2003), Les étincelles du hasard, t. II: Athéisme de l'écriture, Paris, Seuil.

Auwers, J.-M., dir. (2002) [1999], La Bible en français. Guide des traductions courantes, Bruxelles, Lumen vitæ (Connaître la Bible 11/12).

BAKer, M., dir. (2001), Routledge Encyclopedia of Translation Studies, Londres / New York, Routledge.

Barstone, W. (1993), The Poetics of Translation. History, Theory, Practice, New Haven / Londres, Yale University Press.

Benjamin, W. (1997) [allemand 1923], "L'abandon du traducteur. Prolégomènes à la traduction des «Tableaux parisiens» de Charles Baudelaire» / trad. par A. Nouss et L. Lamy, Traduction, terminologie, rédaction, 10/2, p. 13-69.

BOYer, F. (2001), «Introduction », dans F. BOYer, J.-P. Prévost et M. SEVIN, dir., La Bible. Nouvelle traduction, Paris / Montréal, Bayard / Médiaspaul, p. 16-25.

Buber, M. (1936), Die Schrift und ihre Verdeutschung, Berlin, Schocken Verlag.

(1994) [allemand 1936], Scripture and Translation / trad. par L. Rosenwald, Bloomington / Indianapolis, Indiana University Press. (2003) [allemand 1964], "Imitation de Dieu ", Écrits sur la Bible / trad. par D. Meur, Paris, Bayard, p. 187-198.

CORAN (1970) / trad. par Kasimirski, Paris, Garnier-Flammarion.

Derrida, J. (1987), «Des tours de Babel », dans Psyché: inventions de l'autre, Paris, Galilée, p. 203-235. 
Flotow, L. von (2000), "Women, Bibles, Ideologies », Traduction, terminologie, rédaction, 13/1, p. 9-20.

Jasper, D., dir. (1993), Translating Religious Texts, New York, St. Martins' Press.

Korsak, M.P. (1992), At the Start. Genesis Made New. A Translation of the Hebrew Text, New York / Londres, Doubleday.

Ladmiral, J.-L. (1990), «Pour une théologie de la traduction », Traduction, terminologie, rédaction, 3/2, p. 121-138.

Long, L., dir. (2005), Translation and Religion. Holy Untranslatable?, Clevedon / Buffalo / Toronto, Multilingual Matters.

Meschonnic, H. (1999), Poétique du traduire, Lagrasse, Verdier. - (2004), Un coup de Bible dans la philosophie, Paris, Bayard.

Robinson, D. (1992), «The Ascetic Foundations of Western Translatology: Jerome and Augustine », Translation and Literature, 1, p. 3-25.

RosenZweig, F. (1998), "L'écriture et le verbe ", L'écriture, le verbe et autres essais / trad. par J.-L. Evard, Paris, Presses universitaires de France (Philosophie aujourd'hui), p. 87-94.

Scholem, G.G. (1974), «L'achèvement de la traduction de la Bible par Martin Buber", dans Le messianisme juif. Essais sur la spiritualité du judaïsme / trad. par B. Dupuy, Paris, Calmann-Lévy, p. 441-447.

SiBOnY, D. (1997²) [1992], Les trois monothéismes, Paris, Seuil (Points. Essais $348)$.

SNYDER, W.H. (1995), Translation Perspectives, t. VIII : Translation: Religion, Ideology, Politics, Binghamton, Center for Research in Translation, State University of New York.

Steiner, G. (2003), De la Bible à Kafka / trad. par P.E. Dauzat, Paris, Hachette (Littératures).

\section{Résumé}

Le religieux et le sacré sont des notions qui réclament inévitablement leur rattachement à la sphère du transcendant dont la nature rend pour le moins complexe l'élaboration de critères d'évaluation quant aux pratiques inhérentes. Cet article analyse ainsi les obstacles qui se posent devant l'essai d'évaluation critique des traductions bibliques. Outre le niveau textuel et linguistique, toute évaluation doit d'emblée prendre en compte les dimensions théologiques latentes. Il importe de cerner le substrat théologique à l'œuvre dans les positions traductologiques, en ce qui concerne le domaine 
biblique, de même que ce substrat agit dans les cultures sécularisées sous la forme d'un impensé ou d'un investissement de substitution.

Les grandes lignes d'une étude de ce type sont ici d'abord suggérées en envisageant les difficultés que pose l'examen des Bibles confessionnelles et militantes. Puis est abordée la divergence, dans les trois monothéismes, des attitudes traductionnelles en regard de la textualité révélée: judaïsme et islam se rejoignent dans la reconnaissance de la dimension interprétative, tandis que le christianisme vise à l'efficacité dans la transmission du message. Il est enfin fait état de la question du messianisme et de sa compréhension, qui pèse avec la même pertinence dans les orientations traductologiques.

La dernière partie procède à un retour sur la «nouvelle traduction » de la Bible parue chez Bayard/Médiaspaul, avant de conclure sur une défense du concept de "traduction athée » qui articulerait un principe d'altérité dont le texte biblique est le premier véhicule.

\section{Abstract}

The notions of the religious and the sacred both inevitably claim their territory in the sphere of the transcendent, whose nature renders the criteria of evaluation of inherent practices more complex. This article analyzes the obstacles that present themselves when attempting a critical evaluation of biblical translations. Apart from the linguistic and textual levels, all evaluations must take into account the theological dimensions. It is important to discern the theological substrate that is at work in every translation, where the biblical field is concerned, the same way that this substrate plays a role in secularized culture, in the form of an unthought.

The general outline of this type of study is first of all to envision the difficulties in the examination of a confessional or militant Bible. Then the different stands that are taken in translation by all three monotheisms in respect to the revealed text will be studied. Judaism and Islam agree on recognizing an interpretive dimension, whereas Christianity aims for efficiency in the transmission of the message. Finally, the question of the Messiah will be examined, as will be its understanding which is considered equally pertinent in the varied orientations of translations.

The last section will re-examine the "Nouvelle Traduction » version of the Bible, published by Bayard/Médiaspaul, before concluding with a defence of an «atheist translation » concept, thus articulating an otherness that is first carried by the biblical text. 